\title{
Spatial filtering with rough metal-dielectric layered metamaterials
}

\author{
Marcin Stolarek, Piotr Wróbel, Tomasz Stefaniuk, Mateusz Wlazło, Anna Pastuszczak, and Rafał Kotyński \\ Faculty of Physics, University of Warsaw, Pasteura 7, 02-093 Warsaw, Poland
}

Received May 14, 2013; accepted June 25, 2013; published June 30, 2013

\begin{abstract}
Imaging through layered metal-dielectric optical metamaterials may be modeled as linear spatial filtering. We characterize the filtering properties of a thin metal-dielectric-metal multilayer structure evaporated by physical vapor deposition. We present the measurements of transmission spectra, and of surface roughness, as well as the simulation results showing the sensitivity of structure properties to surface roughness. Surface roughness is found to be the major factor that limits the theoretical filtering properties of the structure.
\end{abstract}

Throughout the last decade, modern optics has been revolutionised by the introduction of sub-wavelength structured photonic materials, such as photonic crystals, optical metamaterials, and plasmonic nanostructures.

In this paper our interest is focused on layered metamaterials with sub-wavelength metallic and dielectric (MDM) layers sandwiched in a periodic way. MDM metamaterials find applications in imaging with subwavelength resolution [1], in sub-wavelength focusing [2], far-field imaging of sub-wavelength objects [3], cloaking [4], or design of superabsorbers [5]. They can be designed to achieve such properties as an extreme anisotropy [6], effective-zero permittivity [7], or hyperbolic dispersion [8], negative refraction [9], superprism or supercollimation effects [10], resonant tunnelling [11], etc.

The major limitations in the development of MDM metamaterials are the need to compensate losses resulting from the complex permittivity of metals, and the extreme sensitivity of their optical properties to the permittivity of their components, to their dimensions and to the smoothness of the layer boundaries. Surface roughness is a particular issue, which cannot be entirely removed using currently available evaporation techniques.

In the present paper we discuss the limitations imposed on the spatial filtering properties of PVD-evaporated metamaterials by surface roughness. For the sake of simplicity, we consider a multilayer consisting of just two metallic and one dielectric layer on a substrate. The same MDM can be also seen as a sort of interference filter, however our focus is on spatial filtering rather than frequency filtering.

Transmission through an optically linear layered system [12] can be conveniently treated using the transfer matrix or the scattering matrix approach. Numerical conditioning of the latter is better. This is especially important for the accurate treatment of evanescent waves in sub-diffraction imaging. The components of the scattering matrix represent the reflection and transmission coefficients between some chosen planes of the MDM (See Fig. 1). The properties of MDM are then enclosed in the transfer function, or alternatively in the point spread function of the structure $[13,14]$. We introduce their definitions from the theory of linear shift-invariant systems (LSI), adopted in the past in Fourier optics [15]. Alternatively the point spread function is sometimes defined as a response to a line dipole in the framework of Green's function analysis [16].

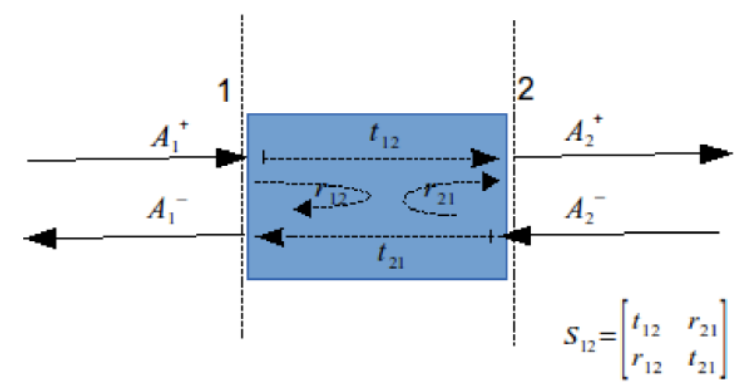

Fig. 1. Graphical interpretation of the scattering matrix.

The scattering matrix $S_{12}$ represents the coupling of amplitudes of counter-propagating waves (or of evanescent waves decaying in opposite directions) at some selected parallel planes 1 and 2 :

$$
\left[\begin{array}{c}
A_{2}^{+} \\
A_{1}^{-}
\end{array}\right]=S_{12}\left[\begin{array}{c}
A_{1}^{+} \\
A_{2}^{-}
\end{array}\right] \text {. }
$$

A larger system consisting of two subsystems with known scattering matrices $S_{12}$ and $S_{23}$, and possibly separated by the distance $d$, is represented by an overall scattering matrix $S_{13}$ which may be derived using Airy's formulas. For instance, the transmission coefficient $t_{13}$ is given as:

$$
t_{13}=\frac{t_{12} t_{23} \exp (i \phi)}{1-r_{21} r_{23} \exp (2 i \phi)}, \quad \phi=k_{z} d
$$


where $k_{z}$ is the wavevector component directed normally to the layer boundaries. Assuming that $k_{z}$ is real and the refractive index at both sides of the structure is the same, the intensity transmission coefficient is equal to $T=\left|t_{13}\right|^{2}$. The amplitude transfer function (ATF, with assumed coherence), is given as $t_{13}\left(v_{x}\right)$, while the incoherent optical transfer function (OTF) is then given as the normalised autocorrelation of ATF [15]. Point spread function (PSF), which is the Fourier Transform of ATF, allows to link the field at the opposite sides of the structure with a simple convolution formula (see Fig. 2).

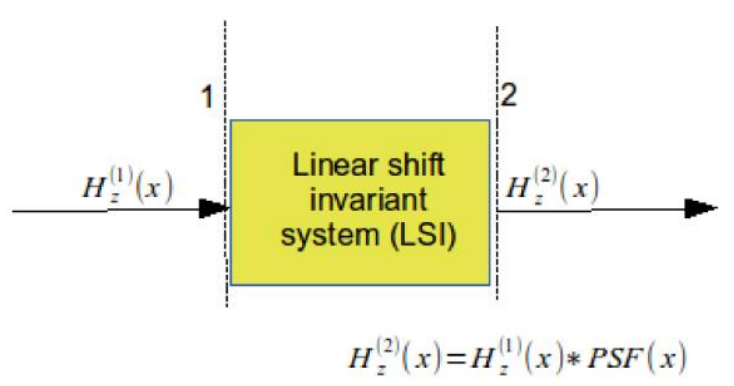

Fig. 2. Schematic of a linear shift invariant system (LSI).

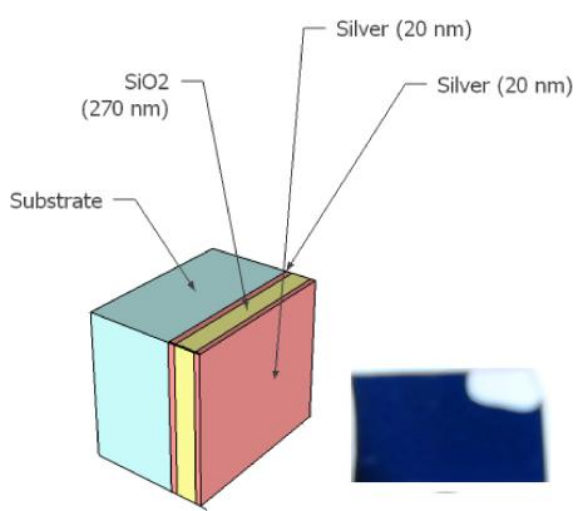

Fig. 3. Schematic and a photograph of the PVD-deposited MDM sample.

We have assumed that the thickness of the multilayer is small with respect to the coherence length $l=\lambda^{2} / \Delta \lambda$. The assumption of coherence is well justified even for LED sources, since a typical layer thickness in optical metamaterials is in the order of tens of nanometers, while the LED coherence length is in the order of tens of micrometers. Notably, the coherence length may be dramatically shortened down to less than $100 \mathrm{~nm}$ if the metallic layers are discontinuous [17]. For a thick layer, i.e. when $d>l / 2 n$ due to spectral averaging, the intensity transmission coefficient takes another form:

$$
T=\left\langle\left|t_{13}\right|^{2}\right\rangle_{\phi}=\frac{\int_{0}^{2 \pi}\left|t_{13}(\phi)\right|^{2} d \phi}{2 \pi}=\frac{\left|t_{12}\right|^{2}\left|t_{23}\right|^{2}}{1-\left|r_{21}\right|^{2}\left|r_{23}\right|^{2}},
$$

where $t_{12}, r_{21}$ refer to transfer through the substrate, while $t_{23}$ and $r_{23}$ refer to the MDM.

The MDM structure which is further analysed is shown in Fig. 3. It consists of a $270 \mathrm{~nm} \mathrm{SiO}_{2}$ layer sandwiched between $20 \mathrm{~nm}$ silver layers and is deposited on a $430 \mu \mathrm{m}$ sapphire substrate. The sample is blue in colour and its transmission spectrum is shown in Fig.4. The surface roughness of the external silver layer is equal to RMS $=5 \mathrm{~nm}$ (see Fig.5), and the surface roughness of the internal layer, which has not been measured for the same sample is in the order of $2 \mathrm{~nm}$. The surface roughness of the substrate is equal to $0.2 \mathrm{~nm}$. In Fig. 6 we show a theoretical transfer function of the MDM for both polarisations, with a visible Fabry-Perot resonance, which changes the spatial filtering character of the MDM from a high-pass filter at $430 \mathrm{~nm}$ to a low-pass filter at 490nm.

In Fig.7 we present finite-difference time-domain (FDTD, open source implementation [18]) simulation results showing the influence of surface roughness on field distribution and, in effect, on filtering properties of the MDM. The RMS and power spectrum of roughness is taken from measurements. We compare the situation without surface roughness, and with measured or estimated surface roughness. The continuous wave superGaussian source with the width FWHM=800nm is positioned in the substrate. Apart from the dominant zeroth spatial frequency, it introduces a range of higher frequencies corresponding to propagating and evanescent waves, sufficient to excite a plasmonic mode on the external Ag layer (see Fig.7a). Surface roughness introduces hot-spots, scattering, affects the overall transmission, and clearly suppresses the plasmonic mode.

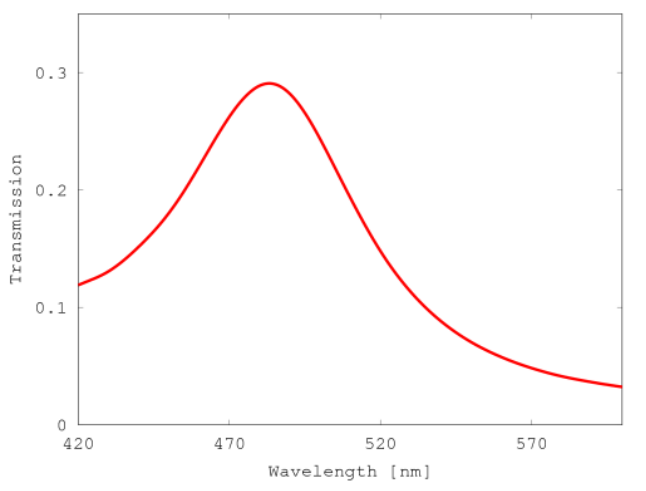

Fig. 4. Transmission spectrum measurement of the MDM

In conclusion, we have discussed the LSI spatial filtering model for layered metamaterials. The model is partly invalidated by the presence of surface roughness. 
We have analysed the spatial filtering properties of a thin metal-dielectric-metal MDM, and we have presented the measurements of transmission spectra, as well as the simulation results showing the sensitivity of structure properties to surface roughness. Surface roughness introduces scattering, produces hot-spots in the near field, decreases transmission, and suppresses the plasmonic mode.

a)
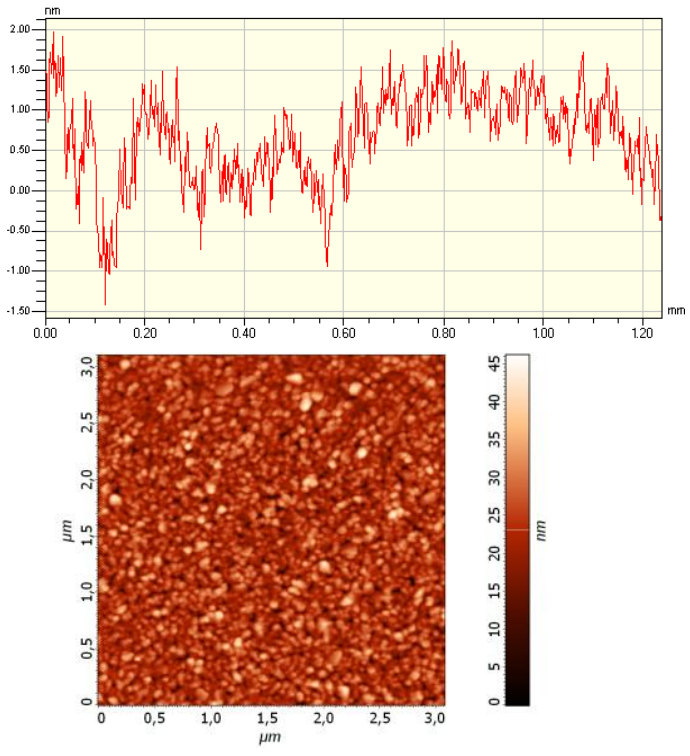

b)

Fig. 5. Surface profile of the multilayer a) measured with non-contact interferometric profilometer, b) and with AFM

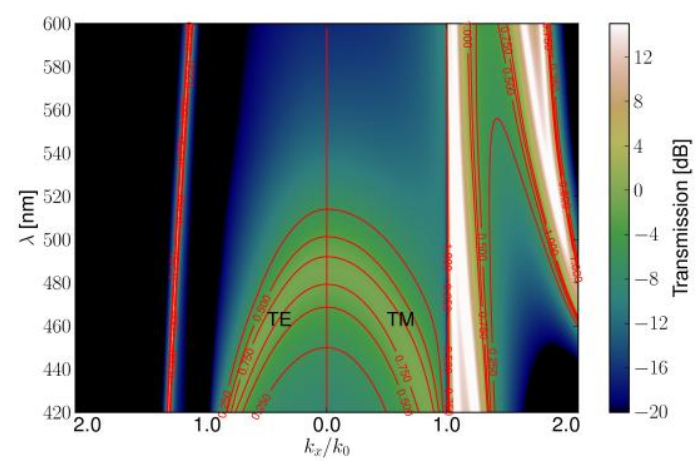

Fig. 6. Theoretical transfer function $|\mathrm{ATF}|^{2}$ of the MDM.

We acknowledge financial support from the Polish National Science Center, grant number UMO2011/01/B/ST3/02281. PL-Grid Infrastructure is acknowledged for providing access to computational resources.
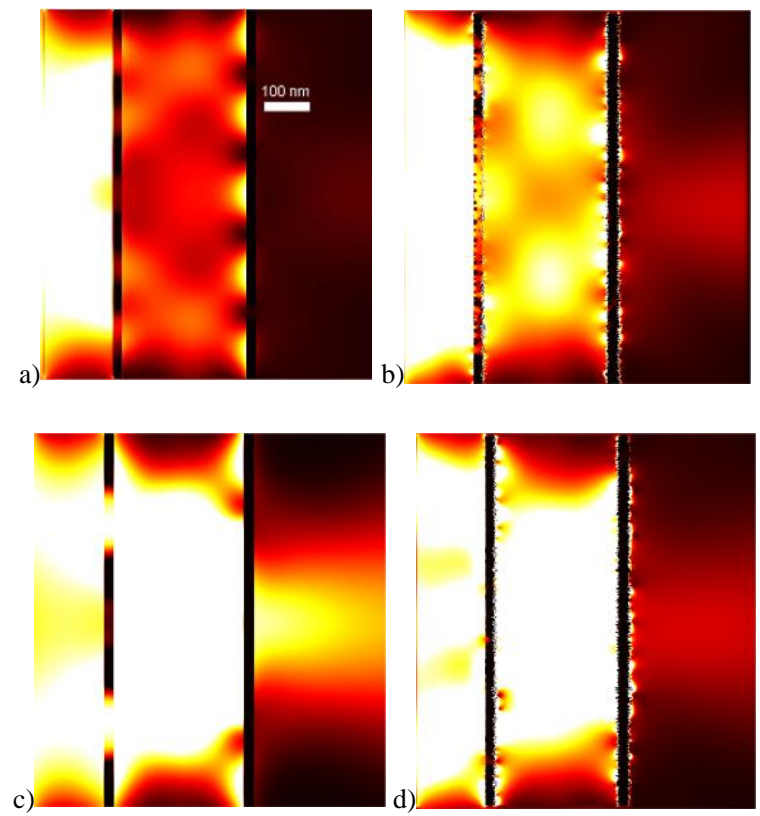

Fig. 7. Energy density calculated with FDTD for the MDM demonstration of the influence of surface roughness on spatial filtering at wavelengths of a, b) $430 \mathrm{~nm}$ and c, d) 490nm; a, c) no surface roughness; $b, d$ ) surface roughness determined experimentally.

\section{References}

[1] N. Fang, H. Lee, C. Sun, X. Zhang, Science 308, 534 (2005).

[2] X. Li, S. He, Y. Jin, Phys. Rev. B 75, 045103 (2007).

[3] Z. Jacob, L.V. Alekseyev, E. Narimanov, Opt. Expr. 14, 8247 (2006).

[4] O. Paul, Y. Urzhumov, C. Elsen, D. Smith, M. Rahm, J. Appl Phys. 111, 123106 (2012).

[5] C. Guclu, S. Campione, and F. Capolino, Phys. Rev. B 86 205130, (2012).

[6] A. Wood, J. B. Pendry, and D. P. Tsai, Phys. Rev. B 74, 115116 (2006).

[7] G. Castaldi, S. Savoia, V. Galdi, A. Alu, N, Engheta, Phys. Rev. B 86, 115123 (2012).

[8] X. Ni, S. Ishii, M.D. Thoreson, V. M. Shalaev, S. Han, S. Lee, A.V. Kildishev, Opt. Expr. 19, 25242 (2011).

[9] M. Scalora, G. D'Aguanno, N. Mattiucci, M.J. Bloemer, D. Ceglia, M. Centini, A. Mandatori, C. Sibilia, N. Akozbek, M.G. Cappeddu, M. Fowler, J. Haus, Opt. Expr. 15, 508 (2007).

[10] D. de Ceglia, M. Vincenti, M. Cappeddu, M. Centini, N. Akozbek, A. D'Orazio, J. Haus, M.J. Bloemer, M. Scalora, Phys. Rev. A, 77, 033848 (2008).

[11] C.-H. Liu and N. Behdad, J. Appl. Phys. 111, 014906 (2012).

[12] Yeh, Optical waves in layered media (Wiley 2005).

[13] N. Mattiucci, D. Aguanno, M. Scalora, M.J. Bloemer, C. Sibilia, Opt. Expr. 17, 17517 (2009).

[14] R. Kotyński, T. Antosiewicz, K. Król, K. Panajotov, J. Opt. Soc. Am. A 28, 111 (2011).

[15] J. Goodman, Introduction to Fourier Optics (Roberts \& Co, 3rd Ed., 2005).

[16] L. Novotny, B. Hecht, Principles of Nano-Optics $\left(2^{\text {nd }}\right.$ ed. Cambridge Univ. Press 2012).

[17] A. Caze, R. Pierrat, R. Carminati, Phys. Rev. Lett. 110, 063903 (2013).

[18] A.F. Oskooi, D. Roundy, M. Ibanescu, P. Bermel, J. Joannopoulos, S.G. Johnson, Comput. Phys. Comm. 181, 687 (2010). 
